\title{
PERSPECTIVE
}

\section{Integrating DNA methylation measures to improve clinical risk assessment: are we there yet? The case of $B R C A 1$ methylation marks to improve clinical risk assessment of breast cancer}

\author{
Ee Ming Wong ${ }^{1,2}$, Melissa C. Southey ${ }^{1,2,3}$ and Mary Beth Terry ${ }^{4,5}$
}

Current risk prediction models estimate the probability of developing breast cancer over a defined period based on information such as family history, non-genetic breast cancer risk factors, genetic information from high and moderate risk breast cancer susceptibility genes and, over the past several years, polygenic risk scores (PRS) from more than 300 common variants. The inclusion of additional data such as PRS improves risk stratification, but it is anticipated that the inclusion of epigenetic marks could further improve model performance accuracy. Here, we present the case for including information on DNA methylation marks to improve the accuracy of these risk prediction models, and consider how this approach contrasts genetic information, as identifying DNA methylation marks associated with breast cancer risk differs inherently according to the source of DNA, approaches to the measurement of DNA methylation, and the timing of measurement. We highlight several DNA-methylation-specific challenges that should be considered when incorporating information on DNA methylation marks into risk prediction models, using BRCA1, a highly penetrant breast cancer susceptibility gene, as an example. Only after careful consideration of study design and DNA methylation measurement will prospective performance of the incorporation of information regarding DNA methylation marks into risk prediction models be valid.

British Journal of Cancer (2020) 122:1133-1140; https://doi.org/10.1038/s41416-019-0720-2

\section{BACKGROUND}

Current cancer risk prediction models are largely grouped based on the extent of family history and genetic data in addition to information relating to non-genetic risk factors such as lifestyle and the environment. Pedigree-based models, which are often used for genetic counselling and risk assessment and for making decisions about chemoprevention and risk-reducing surgeries, ${ }^{1}$ have been improved through the integration of genetic information about high and intermediate penetrant cancer genes. ${ }^{2-4}$ Three such breast cancer models-BOADICEA (Breast and Ovarian Analysis of Disease Incidence and Carrier Estimation Algorithm model), IBIS (the International Breast cancer Intervention Study model) and BRCAPRO - have the ability to predict the probability of carrying a pathogenic breast cancer susceptibility variant in $B R C A 1 / 2$ as well as the absolute risk of developing breast cancer, with higher discrimination for the former than the latter. ${ }^{2-4}$ BOADICEA has also been extended to include information about genetic variation in CHEK2, PALB2 and $A T M{ }^{5}$ as well as polygenic risk scores (PRS), which include hundreds of genetic variants identified from large genome-wide association studies (GWAS)., Although the gain from inclusion of a PRS may be limited overall, there is gain in prediction for women at the extremes of the PRS. In contrast to BOADICEA, IBIS and BRCAPRO, which includes details about the ages of cancer diagnoses in the relatives as well as germline mutations in BRCA1/2, other breast cancer risk models like the commonly used Breast Cancer Risk Assessment Tool (BCRAT) generally consider family history based on the number of first-degree family relatives affected and/or ever/never family history. Most models now include non-genetic established breast cancer risk factors (e.g. parity, age at menarche, hormone use).

Increasingly large-scale studies support that many of the breast cancer predisposition genes can also influence risk when altered through epigenetic mechanisms, such as DNA methylation and histone modification, and that such mechanisms might occur more commonly than currently appreciated. ${ }^{8,9}$ Epigenetic alterations have been demonstrated to be associated with an increased risk of certain diseases, such as Fragile $X$ syndrome, Prader-Willi syndrome, and various cancers, and are increasingly being measured in epidemiological studies. ${ }^{10-12}$ However, potential integration of epigenetic information into risk prediction models requires further prospective evidence that might be more challenging to collect than those studies incorporating genetic information.

Here, we outline the case for the integration of epigenetic measures, in the form of blood-based DNA methylation marks, into existing risk prediction models using the example of $B R C A 1$, the breast cancer susceptibility gene with the highest penetrance,

\footnotetext{
${ }^{1}$ Precision Medicine, School of Clinical Sciences at Monash Health, Monash University, Clayton, VIC, Australia; ${ }^{2}$ Department of Clinical Pathology, The University of Melbourne,

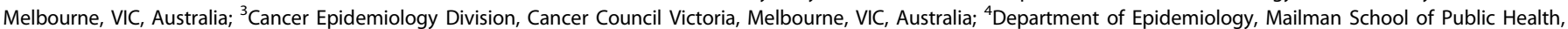
Columbia University, New York, NY, USA and ${ }^{5}$ Herbert Irving Comprehensive Cancer Center, Columbia University, New York, NY, USA Correspondence: Mary Beth Terry (mt146@columbia.edu)
} 
1134

to illustrate the gaps in our knowledge that need to be addressed to improve clinical risk assessment. We selected this gene because the integration of germline mutation status of $B R C A 1$ has already been shown to improve risk assessment and knowledge of the germline $B R C A 1$ status has already altered clinical practice in terms of chemoprevention, recommendations for risk-reducing surgeries, and screening frequency. We consider germline DNA methylation of $B R C A 1$ in all women and not just in women with pathogenic variants in BRCA1. The points we raise would also relate to other epigenetic marks such as histone acetylation and histone methylation.

DNA methylation refers to the addition of a methyl $\left(\mathrm{CH}_{3}\right)$ group to the cytosine residue of a cytosine-guanidine pair in the DNA sequence, commonly referred to as a CpG dinucleotide. DNA methylation is an essential component in early development through a process known as epigenetic reprogramming. ${ }^{13}$ In adult cells, it has been shown to be extensively involved in the initiation and progression of cancer whereby aberrant DNA methylation can lead to silencing and loss of expression of tumour suppressor genes such as BRCA1, MLH1 and $A T M^{14-17}$ and genomic instability. ${ }^{18,19}$ In breast cancer, aberrant DNA methylation levels across specific breast cancer susceptibility genes, such as BRCA $1,{ }^{20-22} A T M{ }^{15,23}$ PALB2 $^{24}$ and Sat $2{ }^{25}$ have been associated with risk of the disease in women with and without pathogenic variants in these genes.

Given that high and intermediate penetrant germline mutation data are now key components to selected risk models like BOADICEA, the potential for improved model performance when extending risk models to include epigenetic markers in the same genes may be great. Several important areas need to be considered before integrating DNA methylation marks into risk assessment models, including the source and nature of the biological material, the approaches used to measure DNA methylation (methodologies and regions of DNA), and the timing of the biological sample collection for DNA methylation measurement, all of which are discussed below using BRCA1 as an example.

\section{BIOLOGICAL MATERIAL FOR DNA METHYLATION ASSESSMENT}

Early studies focused on identifying changes in DNA methylation marks in disease-affected tissues ${ }^{26,27}$ have demonstrated the utility of these changes in further subtyping cancers and refining precision medicine, ${ }^{28-30}$ as well as proving valuable for predicting prognosis after cancer diagnosis. ${ }^{31-33}$ The use of DNA methylation marks for risk prediction, however, often requires the use of surrogate tissue and/or blood-based biomarkers (for review, see refs. ${ }^{9,34}$ ) that can be reliably and repeatedly measured using noninvasive sampling. Here we would like to emphasise that a good predictive marker need not be measured from the potential site of carcinogenesis, e.g. measured in DNA sourced from breast tissue or breast milk to determine breast cancer risk-a good predictive marker needs only to be associated with the disease of interest and to be stable over repeated measurements.

Caution is rightly warranted when using blood-derived DNA modifications as biomarkers. DNA methylation displays cell-typespecific heterogeneity ${ }^{35}$ and, as such, methylation measured in blood-derived DNA is influenced by the proportion of cell types present in the blood sample. To address this, study designs often match case-control pairs by the source of DNA (e.g. whole blood, lymphocyte fraction, buffy coat) and control for variation in blood sample cellularity as part of the analytic process using statistical methods such as that proposed by Houseman et al. ${ }^{36}$ Continued improvement of these statistical methods will further improve the accuracy of cell-type adjustment. Specifically, white blood cells, as a non-invasive source of DNA prior to disease onset, has been used for studies searching for DNA methylation marks that can be useful for understanding cancer susceptibility (the focus of this Perspective).
Using DNA derived from peripheral blood, we reported that constitutional BRCA1 promoter methylation-that is, DNA methylation that is present in every cell of the body-is associated with a 3.5 -fold increased risk (95\% confidence interval [Cl]: 1.4, 10.5) of developing early-onset breast cancer of a specific histological type in non BRCA1 mutation carriers. ${ }^{21}$ Another study conducted in Japan also reported that $B R C A 1$ promoter methylation detected in peripheral blood cells is associated with an increased risk of developing breast cancer (all ages) (odds ratio [OR] 1.73, 95\% Cl: $1.01,2.96) .{ }^{20}$ Methylation of the ATMmvp2a intergenic region and the Sat2 repetitive element have also been reported to be associated with an increased risk of breast cancer (women in the highest quintile OR $1.89,95 \% \mathrm{Cl}: 1.36,2.64$ in peripheral blood, and OR $2.09,95 \% \mathrm{Cl}: 1.09,4.03$ in white blood cells, respectively). ${ }^{23,25} \mathrm{Xu}$ et al. $^{10}$ identified 250 blood-based CpG dinucleotides that were differentially methylated $\left(P_{\text {FalseDiscoveryRate }}<0.05\right)$ between cases and controls, ${ }^{10}$ and that five differentially methylated CpG dinucleotides had similar model discrimination as the BCRAT model that included nine GWAS common variants (area under the curve (AUC) $65.8 \%$, $95 \% \mathrm{Cl}: 61.0,70.5 \%$ versus $66.1 \%, 95 \% \mathrm{Cl}: 61.0,71.3 \%)$.

Several epigenome-wide association studies (EWAS) have also demonstrated an association between global DNA methylation levels and breast cancer risk. ${ }^{11,12}$ However, a 2019 meta-analysis of four EWAS did not find an association between blood-based DNA methylation and the risk of breast cancer; ${ }^{37}$ this result could perhaps be partly explained by the later age of onset in the affected women (further discussed below), or the different analytic approaches used by each study (e.g. cell-type correction, normalisation of raw data and data transformation).

\section{DNA METHYLATION: METHODOLOGY AND MEASUREMENT}

Laboratory methodologies vary and thus measures of DNA methylation cannot, in all instances, be directly compared or easily combined. ${ }^{9,34}$ We will not discuss these methods in detail except to outline in Box 1 the experimental throughput, sensitivity, input DNA requirements and cost-issues that should be considered for epidemiology studies and when evaluating data for integration into cancer risk models. Laboratory methods used to assess DNA methylation can be divided into three main categories: loci-specific; array-based and bisulphite sequencing methods (see Box 1).

Given the different considerations for each of the three methodologies, an approach that targets risk-associated DNA methylation regions, akin to the commonly used gene panel sequencing methods in the germline context, might prove the most useful in integrating new information into cancer risk models. For example, although identified GWAS variants may be used in the future clinically through an integration of a PRS, genetic information from sequencing studies, which include fuller genetic alterations than those included in GWAS, are already being used clinically through gene panel tests. In a similar way, we might expect that studies that include deeper investigation of epigenetic alterations in the same genes used in these gene panels may be clinically more beneficial than information from an EWAS, which only includes selected $\mathrm{CpGs}$ from these high and intermediate penetrant genes.

A meta-analysis found that DNA methylation of the BRCA1 promoter was more common in women diagnosed with breast cancer compared with unaffected women. ${ }^{38}$ A number of different study designs with different methylation markers, including methylation measured in blood-derived DNA and from histologically normal and malignant breast tissues, were included in this meta-analysis. BRCA1 promoter methylation was associated with an increased risk of developing breast cancer (OR 3.15, 95\% Cl: $1.97,5.03, P<0.001$ ), advanced stage histopathology features, and triple-negative disease. ${ }^{38}$ When considering only the nine bloodbased studies, BRCA1 methylation was associated with a 1.87-fold increased breast cancer risk $(95 \% \mathrm{Cl}: 1.19,2.96, P=0.007){ }^{38}$ 
Tang et al. ${ }^{34}$ published a comprehensive review of bloodderived DNA methylation marks associated with an increased risk of breast cancer. Although overall increased BRCA1 promoter methylation was observed in the blood-derived DNA of affected women, the breast cancer risk estimates of the meta-analysis showed considerable variation between studies. $^{34}$ While the factors mentioned above (DNA source, measurement type, methodology) could have contributed to the observed heterogeneity, it is possible that different regions measured even within the same gene, as highlighted by Zhang and Long, ${ }^{38}$ could also provide some explanation for the variation.

Although the majority of studies have assessed blood-derived DNA methylation in the same BRCA1 promoter region (primarily overlapping the bi-directional promoter and transcription start sites), some studies have measured DNA methylation at other loci, such as the CpG island, which is likely to have contributed to the different findings (Table 1; Fig. 1). ${ }^{39,40}$ Our experience with the Infinium HumanMethylation450 BeadChip array, which has been validated using TCGA data, showed that the CpG island (Chromosome17: 41278135-41278459) is highly methylated in blood-derived DNA from both affected and unaffected women and lacks variability between individuals. ${ }^{41,42}$ Therefore, DNA methylation assessment of this invariant region is likely to be uninformative and, indeed, no differences in BRCA1 DNA methylation levels were observed in this region between cases and controls irrespective of their BRCA1 or BRCA2 mutation status. ${ }^{39,40}$ Ziller et al. $^{43}$ found that DNA methylation levels across the intermediate and low $\mathrm{CpG}$ density promoters and transcription start sites, rather than the $\mathrm{CpG}$ islands, are dynamic and variable between individuals. ${ }^{42,43}$ These two observations strongly indicate that the region outside of the $\mathrm{CpG}$ island, rather than the $\mathrm{CpG}$ islands itself, could stand to be more informative for DNA methylation assessment for risk prediction.

Only three CpG dinucleotides in BRCA1 exon 1a $(+8,+14$ and +16) have been evaluated across most studies (12 of the 14 studies listed in Table 1; Fig. 1). However, variability in DNA methylation levels and analysis outcomes across these three sites could still be possible for several reasons as highlighted below. A key aspect that may affect interpretation of the differences across studies is whether or not the study has specifically sampled women with a breast cancer family history, and with few exceptions, ${ }^{44}$ most studies of the association between epigenetic marks and breast cancer risk have been conducted in mainly postmenopausal women. Additionally, inconsistencies in the evidence could be explained by the different methodologies used to assess DNA methylation: seven different methods were used across the 14 studies (Table 1). With the exception of pyrosequencing, bisulphite cloning followed by Sanger sequencing or bisulphite sequencing, methylation-specific methods such as MethylationSensitive High Resolution Melt (MS-HRM) analysis and MethyLight real-time PCR, provide an average methylation value across the interrogated region. Unless all CPG dinucleotides in the region of interest display similar methylation levels, this average methylation value might not enable an association between methylation and breast cancer risk, particularly if only a subset of CpG dinucleotides in the region are relevant to disease risk.

Differences across studies might also be driven by genetic variation, which can influence DNA methylation levels at some genomic regions (known as methylation quantitative trait loci). We previously identified two $B R C A 1$ promoter region genetic variants in blood-derived DNA that were associated with $B R C A 1$ promoter methylation (rs11655505 $(P=0.035)$ and rs799906 $(P=0.017))^{21}$ but the publication of few, if any, reports on genetic variants that are positively associated with BRCA1 promoter methylation rendered these results inconclusive until Evans et al ${ }^{45}$ identified a dominantly inherited BRCA1 $5^{\prime}$ untranslated region variant (NM_007294.3:C.-107 A>T) in two multiple-case breast and ovarian cancer families. ${ }^{45}$ Hemi-methylation of the
Box 1 Laboratory methods to assess DNA methylation

Loci-specific methods include MethyLight Real-time $P C{ }^{46}$ Methylation-Specific $\mathrm{PCR}^{47}$ EpiTYPERMassARRAY System ${ }^{48}$ and pyrosequencing. ${ }^{49}$ Although capable of detecting low levels of methylation (as low as 1\%), these methods are laborious and low-throughput (limited by the number of samples and/or number of regions that can be evaluated in any one assay). Individual assays need to be designed for each region of interest, with each amplicon typically 100-200 base pairs (bp) in size to retain its sensitivity (500 bp for the MassARRAY). DNA of variable quality derived from blood, plasma, dried blood spots and formalin-fixed paraffin-embedded (FFPE) material can be applied to these methods. ${ }^{21,25}$ Locispecific assays are cost-effective and their increased sensitivity makes them suitable for validation studies.

Array-based methods that measure DNA methylation at a large number of CpG dinucleotides across the genome are less sensitive than loci-specific methods particularly at the extremes of DNA methylation ${ }^{50-54}$ although the sensitivity of the Illumina Methylation EPIC array seems to have improved markedly at these extreme DNA methylation levels. ${ }^{52}$ Arrays are suitable candidates for large epidemiology studies and have been instrumental in the success of a large number of EWAS.11,12,37 These arrays specifically assess the most informative regions of the epigenome such as gene promoters, CpG islands, enhancer and regulatory regions. Although relatively cost-effective in the context of throughput and number of evaluated CpG dinucleotides, further validation is often required due to their limited sensitivity. DNA derived from blood, plasma, dried blood spots, saliva, fresh-frozen and FFPE material have all been successfully assayed using this platform. ${ }^{42,55,56}$

Bisulphite sequencing methods include reduced-representation bisulphite sequencing (RRBS), ${ }^{57}$ MethylC-seq, ${ }^{58}$ targeted bisulphite sequencing and whole-genome bisulphite sequencing (WGBS). ${ }^{59}$ These methods quantitatively detect methylation at single base resolution and, depending on the assay, can evaluate methylation at a specific region (e.g. targeted bisulphite sequencing), at CpG-dense areas (e.g. RRBS) or across the methylome(e.g. WGBS).The increased resolution of these techniques also enables the detection of technical variabilities not previously possible using lower resolution techniques. The community's experience with WGBS is mixed: areas of variable coverage and bisulphite conversion inefficiencies within and between, samples have, in many instances, affected data interpretation. It should also be noted that, of the 28 million CpG dinucleotides across the methylome, $70-80 \%$ are stably methylated and therefore are uninformative across different cell types. ${ }^{43}$ Bisulphite sequencing methods are the most expensive of the laboratory methods and generally require high molecular weight DNA, which can be challenging for many epidemiology studies that only have access to limited quantities of often degraded DNA derived from field-collected bioresources. Additionally, as sample size increases, the common set of $\mathrm{CpGs}$ with quality data after an experiment may result in a matrix containing similar or fewer CpGs than an array-based approach.

$B R C A 1$ promoter region was found soma-wide, including in leukocyte-derived DNA in heterozygote carriers with BRCA1 promoter methylation associated with the c. $-107 \mathrm{~A}>\mathrm{T}$ variant, explaining at least $1.25 \%$ of $B R C A 1$ pathogenic variants in their multiple-case breast and ovarian cancer families (Table 1).

\section{TIMING OF SAMPLE COLLECTION FOR DNA METHYLATION MEASUREMENT}

Measuring DNA methylation marks requires considerable attention to the hypothesis being tested to not only define the relevant target tissue, but to also ascertain the appropriate timing of the biological sample collection. Blood-based DNA methylation marks can be altered by intrauterine exposures (e.g. prenatal famine ${ }^{60}$ ) as well as environmental conditions later in life (e.g. air pollution $^{61,62}$ ). Alterations in DNA methylation might hold great potential as surrogate markers for factors that are not easily measured by questionnaires-for instance, Boyne et al. ${ }^{62}$ and Johansson et al. ${ }^{63}$ have found that long-term hormonal exposure can be proxied through DNA methylation signatures.

However, although the potential for the use of DNA methylation markers is great, there are also many challenges that need to be overcome for DNA methylation measures to make a significant contribution to breast cancer risk prediction models. The key considerations include making the appropriate measurements and then interpreting these measurements, especially those that depend on time cognisant of the research question. Conducting GWAS and measuring common genetic variation in large 


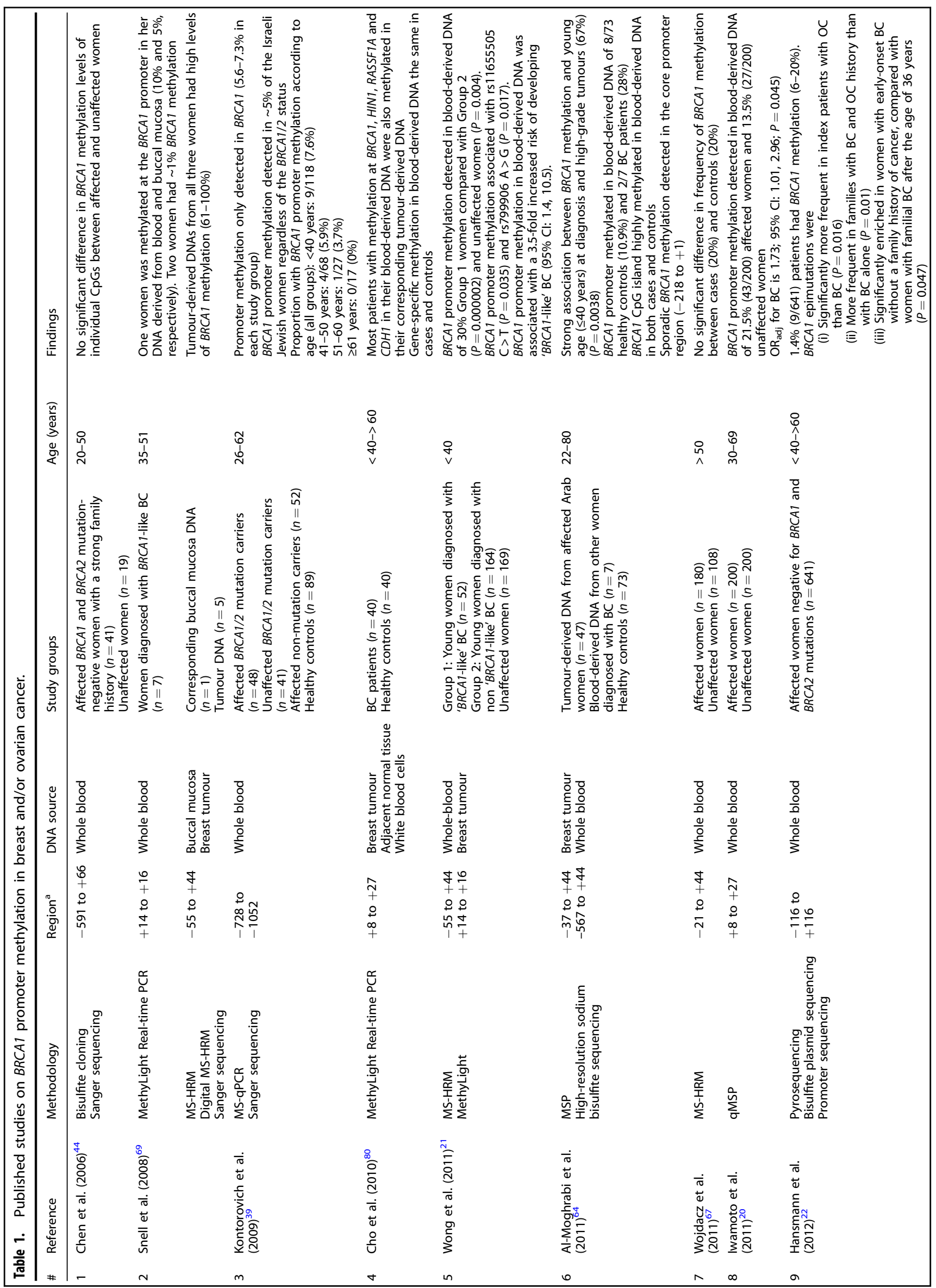




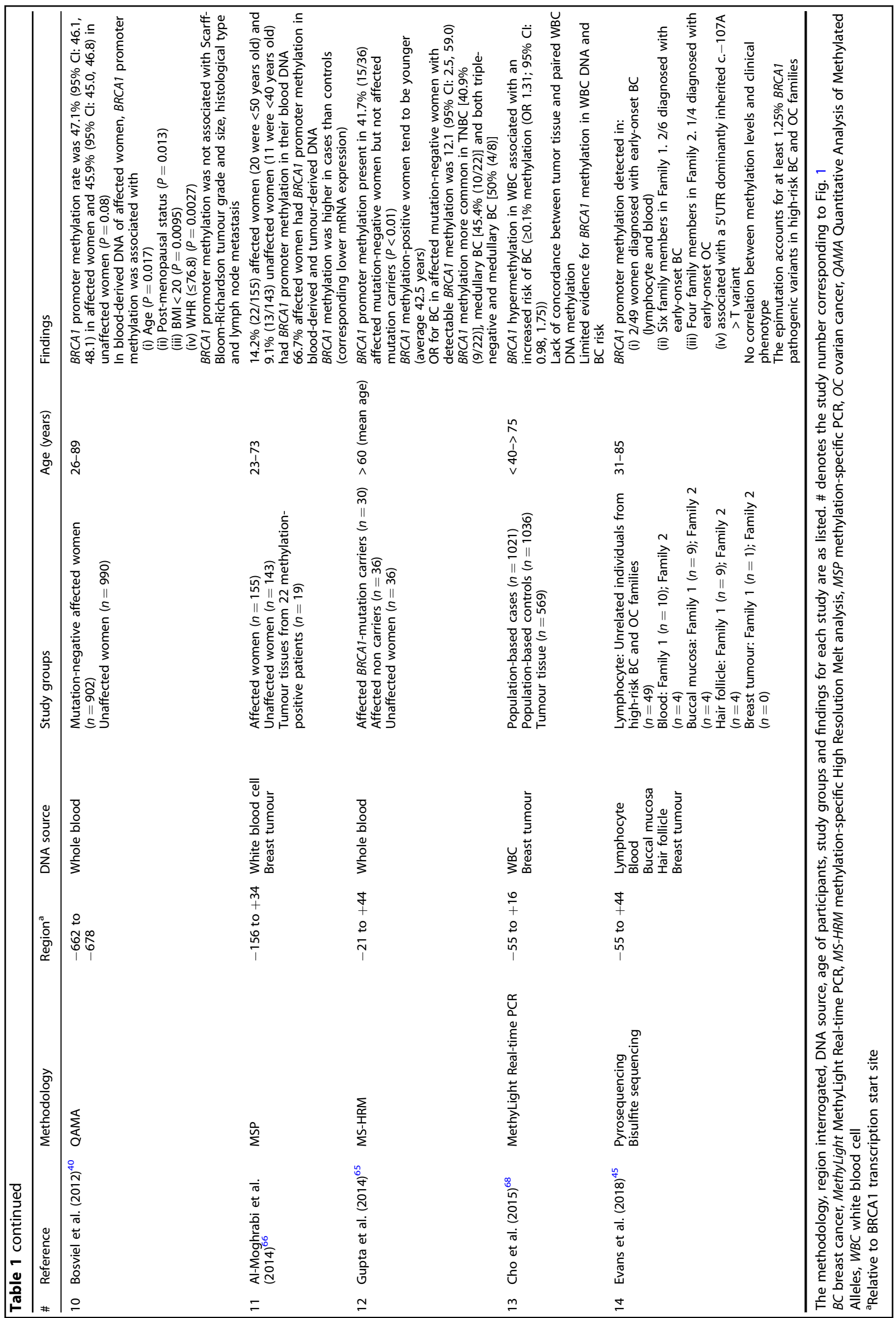




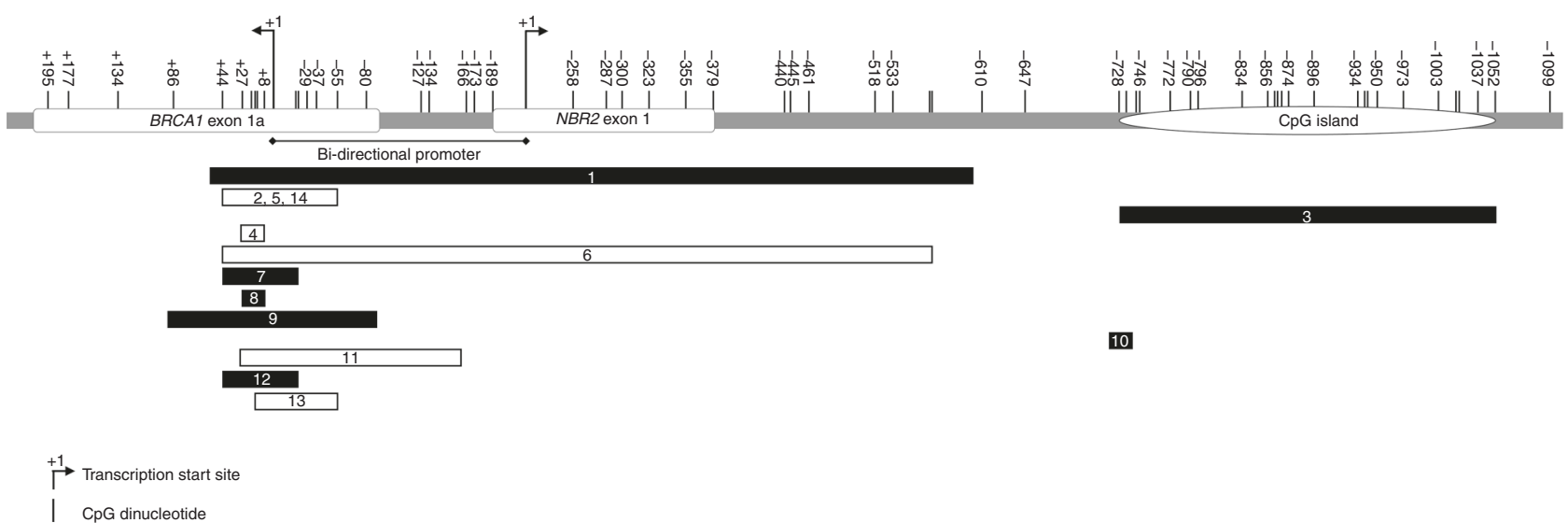

Fig. 1 The BRCA1 promoter regions assessed for methylation by the studies listed in Table 1. The number(s) in each bar corresponds to the study number (\#) in Table 1. The BRCA1 promoter region assessed by each study is represented by a horizontal bar. Black bars represent studies that measured BRCA1 promoter methylation in blood-derived DNA. White bars represent studies that measured BRCA1 promoter methylation in blood-derived DNA and DNA derived from sources other than blood. Each CpG dinucleotide is represented as a vertical line and numbered relative to the BRCA1 transcription start site (denoted by +1 ).

consortia, by contrast, have generally been straightforward, as genes do not change with time, DNA can be collected from a variety of sources, and the type of genotyping assay does not usually affect the aggregating of data. However, exposure measurements in large consortia are often limited to a single time point, and repeated measurements of lifestyle and environmental exposures are rarely available. Unfortunately, this also applies to studies trying to identify DNA methylation risk factors for disease, which may be just as important if not more so than genetic (GWAS-discovered) variants in the same gene. ${ }^{10-12}$

Changes in DNA methylation over time can also affect the interpretation of data collected across different participant age groups. In Table 1, studies include individuals in different age groups, with the larger studies weighted towards older, averagerisk individuals. Associations between BRCA1 DNA methylation and breast cancer have more commonly been reported by studies that specifically examined BRCA1 promoter methylation in younger women ( $<45$ years) or stratified their findings by age at diagnosis. ${ }^{21,22,64-66}$ When we specifically sampled for earlyonset breast cancer cases ( $<40$ years), the association with BRCA1 promoter methylation and breast cancer risk is much stronger (OR 3.5, 95\% Cl: 1.4,10.5). ${ }^{21}$ Wodjacz et al. ${ }^{67}$ measured $B R C A 1$ methylation in post-menopausal women and failed to find any significant differences between cases and controls. Cho and colleagues $^{68}$ assessed BRCA1 promoter methylation in women across a wide age range ( $<45$ to $>75$ years old) but did not consider a sub-analysis of young women, which is particularly relevant as BRCA1 promoter methylation is more frequently detected in young women diagnosed with breast tumours and with a specific histological type. ${ }^{21,66,69}$ If the breast cancer risk associated with BRCA1 promoter methylation mimics germline pathogenic variants (where the incidence of breast cancer in $B R C A 1$ mutation carriers rises rapidly after the age of 30 years $^{70}$ ) the timing of DNA sampling and the case mix in terms of age and absolute risk in studies of DNA methylation will likely affect the relative risk estimates for methylation markers and breast cancer.

Finally, epigenetic aging should also be taken into account (biological age based on the methylation measurement of specific sets of (pG dinucleotides) as it has been shown to be associated with risk of mortality and disease..$^{71-73}$ Using a prospective study design and three epigenetic clocks (Horvath, Hannum and Levine), ${ }^{74-76}$ Kresovich et al. ${ }^{77}$ found that a 5 -year acceleration of epigenetic age (defined as the difference between the biological age and chronological age) was associated with increased risk for breast cancer (hazard ratio [HR]: 1.08-1.15,
95\% Cl: $1.00-1.23, P<0.001-0.04)$. The inclusion of data on epigenetic age acceleration might therefore be pertinent to increase the accuracy of breast cancer risk prediction models.

\section{CONCLUSIONS}

Extensive efforts to identify DNA methylation marks associated with risk of breast cancer have so far identified a small number of potential DNA methylation marks of interest with the prime example being BRCA1. We have outlined a number of issues for consideration before information about DNA methylation can be integrated into breast cancer risk prediction models. We used the example of $B R C A 1$ to illustrate the challenges faced when considering DNA methylation changes in a breast cancer susceptibility gene, as well as to caution that even when considering single-gene studies, results can be heterogeneous depending on study selection, sampling and laboratory methods.

With management of the above concerns, this line of research has several strengths, including the prevalence of DNA methylation changes (which is often higher than that of genetic changes), the potential magnitude of the association between DNA methylation and cancer risk, and DNA methylation alterations reflecting in part changes in exposures and conditions during the course of life. These three factors combined suggest that overcoming the challenges in conducting studies and implementing measurements in the clinic might be outweighed by the gain in improved accuracy of risk prediction models and ultimately the more precise identification of individuals who can benefit most from early intervention and early detection. As an example, there is an increasing incidence of advanced breast cancer in young women under the age of 40 in the USA ${ }^{78}$ and, globally, breast cancer remains the top cancer in terms of both incidence and mortality in women in most countries across continents ${ }^{79}$ additional methods are needed to identify these high-risk women. It is in this context that measures of DNA methylation, particularly for DNA methylation alterations in high and intermediate penetrant breast cancer susceptibility genes, combined with germline genetic testing of cancer susceptibility genes might identify young women who are at increased risk with more precision than current approaches and models. Women identified as being at high risk via these multi-omic approaches might benefit from supplemental screening modalities such as MRI. Incorporation of information regarding DNA methylation changes, including those that target high and intermediate penetrant breast cancer susceptibility genes, into risk assessment may 
therefore prove important in ages and settings where populationbased screening by mammography is not possible.

\section{AUTHOR CONTRIBUTIONS}

M.B.T. conceived the idea for the manuscript. E.M.W. and M.B.T. drafted the first version of the manuscript. M.C.S. contributed to manuscript writing. All authors reviewed and approved the final manuscript.

\section{ADDITIONAL INFORMATION}

Ethics approval and consent to participate Not applicable

Consent to publish Not applicable

Data availability No data were generated for this manuscript.

Competing interests The authors declare no competing interests.

Funding information E.M.W. is a recipient of the Dyason Fellowship. M.C.S. is a NHMRC Senior Research Fellow. M.B.T. is supported by the Breast Cancer Research Foundation.

Publisher's note Springer Nature remains neutral with regard to jurisdictional claims in published maps and institutional affiliations.

\section{REFERENCES}

1. Terry, M. B., Liao, Y., Whittemore, A. S., Leoce, N., Buchsbaum, R., Zeinomar, N. et al. 10-year performance of four models of breast cancer risk: a validation study. Lancet Oncol. 20, 504-517 (2019).

2. Antoniou, A. C., Pharoah, P. D., McMullan, G., Day, N. E., Stratton, M. R., Peto, J. et al. A comprehensive model for familial breast cancer incorporating BRCA1, BRCA2 and other genes. Br. J. Cancer 86, 76-83 (2002).

3. Berry, D. A., Parmigiani, G., Sanchez, J., Schildkraut, J. \& Winer, E. Probability of carrying a mutation of breast-ovarian cancer gene BRCA1 based on family history. J. Natl Cancer Inst. 89, 227-238 (1997).

4. Tyrer, J., Duffy, S. W. \& Cuzick, J. A breast cancer prediction model incorporating familial and personal risk factors. Stat. Med. 23, 1111-1130 (2004).

5. Lee, A. J., Cunningham, A. P., Tischkowitz, M., Simard, J., Pharoah, P. D., Easton, D. F. et al. Incorporating truncating variants in PALB2, CHEK2, and ATM into the BOADICEA breast cancer risk model. Genet. Med. 18, 1190-1198 (2016).

6. Mavaddat, N., Michailidou, K., Dennis, J., Lush, M., Fachal, L., Lee, A. et al. Polygenic risk scores for prediction of breast cancer and breast cancer subtypes. Am. J. Hum. Genet. 104, 21-34 (2019).

7. Khera, A. V., Chaffin, M., Aragam, K. G., Haas, M. E., Roselli, C., Choi, S. H. et al. Genome-wide polygenic scores for common diseases identify individuals with risk equivalent to monogenic mutations. Nat. Genet. 50, 1219-1224 (2018).

8. Widschwendter, M., Jones, A., Evans, I., Reisel, D., Dillner, J., Sundström, K. et al. Epigenome-based cancer risk prediction: rationale, opportunities and challenges. Nat. Rev. Clin. Oncol. 15, 292 (2018).

9. Terry, M. B., McDonald, J. A., Wu, H. C., Eng, S. \& Santella, R. M. epigenetic biomarkers of breast cancer risk: across the breast cancer prevention continuum. Adv. Exp. Med. Biol. 882, 33-68 (2016).

10. Xu, Z., Bolick, S. C., DeRoo, L. A., Weinberg, C. R., Sandler, D. P. \& Taylor, J. A. Epigenome-wide association study of breast cancer using prospectively collected sister study samples. J. Natl Cancer Inst. 105, 694-700 (2013).

11. Severi, G., Southey, M. C., English, D. R., Jung, C. H., Lonie, A., McLean, C. et al. Epigenome-wide methylation in DNA from peripheral blood as a marker of risk for breast cancer. Breast Cancer Res. Treat. 148, 665-673 (2014).

12. van Veldhoven, K., Polidoro, S., Baglietto, L., Severi, G., Sacerdote, C., Panico, S. et al. Epigenome-wide association study reveals decreased average methylation levels years before breast cancer diagnosis. Clin. Epigenetics 7, 67 (2015).

13. Cantone, I. \& Fisher, A. G. Epigenetic programming and reprogramming during development. Nat. Struct. Mol. Biol. 20, 282-289 (2013).

14. Rice, J. C., Massey-Brown, K. S. \& Futscher, B. W. Aberrant methylation of the BRCA1 CpG island promoter is associated with decreased BRCA1 mRNA in sporadic breast cancer cells. Oncogene 17, 1807-1812 (1998).

15. Flanagan, J. M., Munoz-Alegre, M., Henderson, S., Tang, T., Sun, $\mathrm{P}$, Johnson, N. et al. Gene-body hypermethylation of ATM in peripheral blood DNA of bilateral breast cancer patients. Hum. Mol. Genet. 18, 1332-1342 (2009).
16. Pinto, D., Pinto, C., Guerra, J., Pinheiro, M., Santos, R., Vedeld, H. M. et al. Contribution of MLH1 constitutional methylation for Lynch syndrome diagnosis in patients with tumor MLH1 downregulation. Cancer Med. 7, 433-444 (2018).

17. Ward, R. L., Dobbins, T., Lindor, N. M., Rapkins, R. W. \& Hitchins, M. P. Identification of constitutional MLH1 epimutations and promoter variants in colorectal cancer patients from the Colon Cancer Family Registry. Genet Med. 15, 25-35 (2013).

18. Kulis, M. \& Esteller, M. DNA methylation and cancer. Adv. Genet. 70, 27-56 (2010).

19. Jones, P. A. \& Baylin, S. B. The epigenomics of cancer. Cell 128, 683-692 (2007).

20. Iwamoto, T., Yamamoto, N., Taguchi, T., Tamaki, Y. \& Noguchi, S. BRCA1 promoter methylation in peripheral blood cells is associated with increased risk of breast cancer with BRCA1 promoter methylation. Breast Cancer Res. Treat. 129, 69-77 (2011).

21. Wong, E. M., Southey, M. C., Fox, S. B., Brown, M. A., Dowty, J. G., Jenkins, M. A et al. Constitutional methylation of the BRCA1 promoter is specifically associated with BRCA1 mutation-associated pathology in early-onset breast cancer. Cancer Prev. Res. (Phila) 4, 23-33 (2011).

22. Hansmann, T., Pliushch, G., Leubner, M., Kroll, P., Endt, D., Gehrig, A. et al. Constitutive promoter methylation of BRCA1 and RAD51C in patients with familial ovarian cancer and early-onset sporadic breast cancer. Hum. Mol. Genet 21, 4669-4679 (2012).

23. Brennan, K., Garcia-Closas, M., Orr, N., Fletcher, O., Jones, M., Ashworth, A. et al. Intragenic ATM methylation in peripheral blood DNA as a biomarker of breast cancer risk. Cancer Res. 72, 2304-2313 (2012).

24. Potapova, A., Hoffman, A. M., Godwin, A. K., Al-Saleem, T. \& Cairns, P. Promoter hypermethylation of the PALB2 susceptibility gene in inherited and sporadic breast and ovarian cancer. Cancer Res. 68, 998-1002 (2008).

25. Wu, H. C., Delgado-Cruzata, L., Flom, J. D., Perrin, M., Liao, Y., Ferris, J. S. et al. Repetitive element DNA methylation levels in white blood cell DNA from sisters discordant for breast cancer from the New York site of the Breast Cancer Family Registry. Carcinogenesis 33, 1946-1952 (2012).

26. Dobrovic, A. \& Simpfendorfer, D. Methylation of the BRCA1 gene in sporadic breast cancer. Cancer Res. 57, 3347-3350 (1997).

27. Bianco, T., Chenevix-Trench, G., Walsh, D. C., Cooper, J. E. \& Dobrovic, A. Tumourspecific distribution of BRCA1 promoter region methylation supports a pathogenetic role in breast and ovarian cancer. Carcinogenesis 21, 147-151 (2000).

28. Rodriguez-Paredes, M. \& Esteller, M. Cancer epigenetics reaches mainstream oncology. Nat. Med. 17, 330-339 (2011).

29. Szyf, M. DNA methylation signatures for breast cancer classification and prog nosis. Genome Med. 4, 26 (2012).

30. Kondrashova, O., Topp, M., Nesic, K., Lieschke, E., Ho, G. Y., Harrell, M. I. et al. Methylation of all BRCA1 copies predicts response to the PARP inhibitor rucaparib in ovarian carcinoma. Nat. Commun. 9, 3970 (2018).

31. Fackler, M. J., Umbricht, C. B., Williams, D., Argani, P., Cruz, L. A., Merino, V. F. et al. Genome-wide methylation analysis identifies genes specific to breast cancer hormone receptor status and risk of recurrence. Cancer Res. 71, 6195-6207 (2011).

32. Stirzaker, C., Zotenko, E., Song, J. Z., Qu, W., Nair, S. S., Locke, W. J. et al. Methylome sequencing in triple-negative breast cancer reveals distinct methylation clusters with prognostic value. Nat. Commun. 6, 5899 (2015).

33. Zhang, S., Wang, Y., Gu, Y., Zhu, J., Ci, C., Guo, Z. et al. Specific breast cancer prognosis-subtype distinctions based on DNA methylation patterns. Mol. Oncol. 12, 1047-1060 (2018).

34. Tang, Q., Cheng, J., Cao, X., Surowy, H. \& Burwinkel, B. Blood-based DNA methylation as biomarker for breast cancer: a systematic review. Clin. Epigenetics 8, 115 (2016)

35. Adalsteinsson, B. T., Gudnason, H., Aspelund, T., Harris, T. B., Launer, L. J., Eiriksdottir, G. et al. Heterogeneity in white blood cells has potential to confound DNA methylation measurements. PLoS ONE 7, e46705 (2012).

36. Houseman, E. A., Accomando, W. P., Koestler, D. C., Christensen, B. C., Marsit, C. J., Nelson, $\mathrm{H}$. H. et al. DNA methylation arrays as surrogate measures of cell mixture distribution. BMC Bioinforma. 13, 86 (2012).

37. Bodelon, C., Ambatipudi, S., Dugue, P. A., Johansson, A., Sampson, J. N., Hicks, B. et al. Blood DNA methylation and breast cancer risk: a meta-analysis of four prospective cohort studies. Breast Cancer Res. 21, 62 (2019).

38. Zhang, L. \& Long, X. Association of BRCA1 promoter methylation with sporadic breast cancers: evidence from 40 studies. Sci Rep. 5, 17869 (2015).

39. Kontorovich, T., Cohen, Y., Nir, U. \& Friedman, E. Promoter methylation patterns of ATM, ATR, BRCA1, BRCA2 and P53 as putative cancer risk modifiers in Jewish BRCA1/BRCA2 mutation carriers. Breast Cancer Res. Treat. 116, 195-200 (2009).

40. Bosviel, R., Garcia, S., Lavediaux, G., Michard, E., Dravers, M., Kwiatkowski, F. et al. BRCA1 promoter methylation in peripheral blood DNA was identified in sporadic breast cancer and controls. Cancer Epidemiol. 36, e177-e182 (2012).

41. Scott, C. M., Wong, E. M., Joo, J. E., Dugue, P. A., Jung, C. H., O'Callaghan, N. et al. Genome-wide DNA methylation assessment of 'BRCA1-like' early-onset breast cancer: Data from the Australian Breast Cancer Family Registry. Exp. Mol. Pathol. 105, 404-410 (2018). 
42. Wong, E. M., Joo, J. E., McLean, C. A., Baglietto, L., English, D. R., Severi, G. et al. Tools for translational epigenetic studies involving formalin-fixed paraffinembedded human tissue: applying the Infinium HumanMethyation450 Beadchip assay to large population-based studies. BMC Res. Notes 8, 543 (2015).

43. Ziller, M. J., Gu, H., Muller, F., Donaghey, J., Tsai, L. T., Kohlbacher, O. et al. Charting a dynamic DNA methylation landscape of the human genome. Nature $\mathbf{5 0 0}$, 477-481 (2013).

44. Chen, Y., Toland, A. E., McLennan, J., Fridlyand, J., Crawford, B., Costello, J. F. et al. Lack of germ-line promoter methylation in BRCA1-negative families with familial breast cancer. Genet. Test 10, 281-284 (2006).

45. Evans, D. G. R., van Veen, E. M., Byers, H. J., Wallace, A. J., Ellingford, J. M., Beaman, G. et al. A dominantly inherited 5' UTR variant causing methylation-associated silencing of BRCA1 as a cause of breast and ovarian cancer. Am. J. Hum. Genet 103, 213-220 (2018).

46. Trinh, B. N., Long, T. I. \& Laird, P. W. DNA methylation analysis by MethyLight technology. Methods 25, 456-462 (2001).

47. Herman, J. G., Graff, J. R., Myohanen, S., Nelkin, B. D. \& Baylin, S. B. Methylationspecific PCR: a novel PCR assay for methylation status of CpG islands. Proc. Natl Acad. Sci. USA 93, 9821-9826 (1996).

48. Ehrich, M., Nelson, M. R., Stanssens, P., Zabeau, M., Liloglou, T., Xinarianos, G. et al. Quantitative high-throughput analysis of DNA methylation patterns by basespecific cleavage and mass spectrometry. Proc. Natl Acad. Sci. USA 102, 15785-15790 (2005)

49. Tost, J., Dunker, J. \& Gut, I. G. Analysis and quantification of multiple methylation variable positions in CpG islands by Pyrosequencing. Biotechniques 35, 152-156 (2003).

50. Sandoval, J., Heyn, H., Moran, S., Serra-Musach, J., Pujana, M. A., Bibikova, M. et al. Validation of a DNA methylation microarray for $450,000 \mathrm{CpG}$ sites in the human genome. Epigenetics 6, 692-702 (2011).

51. Dedeurwaerder, S., Defrance, M., Calonne, E., Denis, H., Sotiriou, C. \& Fuks, F. Evaluation of the Infinium Methylation 450K technology. Epigenomics 3, 771-784 (2011).

52. Pidsley, R., Zotenko, E., Peters, T. J., Lawrence, M. G., Risbridger, G. P., Molloy, $P$. et al. Critical evaluation of the Illumina MethylationEPIC BeadChip microarray for whole-genome DNA methylation profiling. Genome Biol 17, 208 (2016).

53. Dugue, P. A., English, D. R., MacInnis, R. J., Joo, J. E., Jung, C. H. \& Milne, R. L. The repeatability of DNA methylation measures may also affect the power of epigenome-wide association studies. Int. J. Epidemiol. 44, 1460-1461 (2015).

54. De Meyer, T., Bady, P., Trooskens, G., Kurscheid, S., Bloch, J., Kros, J. M. et al. Genomewide DNA methylation detection by MethylCap-seq and Infinium HumanMethylation450 BeadChips: an independent large-scale comparison. Sci. Rep. 5, 15375 (2015).

55. Joo, J. E., Wong, E. M., Baglietto, L., Jung, C. H., Tsimiklis, H., Park, D. J. et al. The use of DNA from archival dried blood spots with the Infinium HumanMethylation450 array. BMC Biotechnol. 13, 23 (2013).

56. Bruinsma, F. J., Joo, J. E., Wong, E. M., Giles, G. G. \& Southey, M. C. The utility of DNA extracted from saliva for genome-wide molecular research platforms. BMC Res. Notes 11, 8 (2018)

57. Meissner, A., Gnirke, A., Bell, G. W., Ramsahoye, B., Lander, E. S. \& Jaenisch, R. Reduced representation bisulfite sequencing for comparative high-resolution DNA methylation analysis. Nucleic Acids Res. 33, 5868-5877 (2005).

58. Urich, M. A., Nery, J. R., Lister, R., Schmitz, R. J. \& Ecker, J. R. MethylC-seq library preparation for base-resolution whole-genome bisulfite sequencing. Nat. Protoc. 10, 475-483 (2015).

59. Lister, R., Pelizzola, M., Dowen, R. H., Hawkins, R. D., Hon, G., Tonti-Filippini, J. et al. Human DNA methylomes at base resolution show widespread epigenomic differences. Nature 462, 315-322 (2009).

60. Heijmans, B. T., Tobi, E. W., Stein, A. D., Putter, H., Blauw, G. J., Susser, E. S. et al. Persistent epigenetic differences associated with prenatal exposure to famine in humans. Proc. Natl Acad. Sci. USA 105, 17046-17049 (2008).

61. Plusquin, M., Guida, F., Polidoro, S., Vermeulen, R., Raaschou-Nielsen, O., Campanella, G. et al. DNA methylation and exposure to ambient air pollution in two prospective cohorts. Environ. Int. 108, 127-136 (2017).

62. Boyne, D. J., Friedenreich, C. M., McIntyre, J. B., Stanczyk, F. Z., Courneya, K. S. \& King, W. D. Endogenous sex hormone exposure and repetitive element DNA methylation in healthy postmenopausal women. Cancer Causes Control 28, 1369-1379 (2017).

63. Johansson, A., Palli, D., Masala, G., Grioni, S., Agnoli, C., Tumino, R. et al. Epigenomewide association study for lifetime estrogen exposure identifies an epigenetic signature associated with breast cancer risk. Clin. Epigenetics 11, 66 (2019).
64. Al-Moghrabi, N., Al-Qasem, A. J. \& Aboussekhra, A. Methylation-related mutations in the BRCA1 promoter in peripheral blood cells from cancer-free women. Int. J. Oncol. 39, 129-135 (2011).

65. Gupta, S., Jaworska-Bieniek, K., Narod, S. A., Lubinski, J., Wojdacz, T. K. \& Jakubowska, A. Methylation of the BRCA1 promoter in peripheral blood DNA is associated with triple-negative and medullary breast cancer. Breast Cancer Res. Treat. 148, 615-622 (2014).

66. Al-Moghrabi, N., Nofel, A., Al-Yousef, N., Madkhali, S., Bin Amer, S. M., Alaiya, A. et al. The molecular significance of methylated BRCA1 promoter in white blood cells of cancer-free females. BMC Cancer 14, 830 (2014).

67. Wojdacz, T. K., Thestrup, B. B., Cold, S., Overgaard, J. \& Hansen, L. L. No difference in the frequency of locus-specific methylation in the peripheral blood DNA of women diagnosed with breast cancer and age-matched controls. Future Oncol. 7, 1451-1455 (2011)

68. Cho, Y. H., McCullough, L. E., Gammon, M. D., Wu, H. C., Zhang, Y. J., Wang, Q. et al. Promoter hypermethylation in white blood cell DNA and breast cancer risk. J. Cancer 6, 819-824 (2015).

69. Snell, C., Krypuy, M., Wong, E. M., Loughrey, M. B. \& Dobrovic, A. BRCA1 promoter methylation in peripheral blood DNA of mutation negative familial breast cancer patients with a BRCA1 tumour phenotype. Breast Cancer Res. 10, R12 (2008).

70. Dite, G. S., Jenkins, M. A., Southey, M. C., Hocking, J. S., Giles, G. G., McCredie, M. R. et al. Familial risks, early-onset breast cancer, and BRCA1 and BRCA2 germline mutations. J. Natl Cancer Inst. 95, 448-457 (2003).

71. Zheng, Y. J. B. T., Colicino, E., Liu, L., Zhang, W., Dai, Q., Shrubsole, M. J., Kibbe, W. A., Gao, T., Zhang, Z., Jafari, N., Vokonas, P., Schwartz, J., Baccarelli, A. A. \& HouL Blood epigenetic age may predict cancer incidence and mortality. EBioMedicine $\mathbf{5}$, 68-73 (2016).

72. Gensous, N., Bacalini, M. G., Pirazzini, C., Marasco, E., Giuliani, C., Ravaioli, F. et al. The epigenetic landscape of age-related diseases: the geroscience perspective. Biogerontology 18, 549-559 (2017).

73. Peter, D., Fransquet, J. W., Robyn, L., Woods, Michael, E., Ernst \& Ryan, Joanne The epigenetic clock as a predictor of disease and mortality risk: a systematic review and meta-analysis. Clin. Epigenetics 11, 62 (2019).

74. Horvath, S. DNA methylation age of human tissues and cell types. Genome Biol. 14, R115 (2013)

75. Hannum, G., Guinney, J., Zhao, L., Zhang, L., Hughes, G., Sadda, S. et al. Genomewide methylation profiles reveal quantitative views of human aging rates. Mol Cell 49, 359-367 (2013).

76. Levine, M. E., Lu, A. T., Quach, A., Chen, B. H., Assimes, T. L., Bandinelli, S. et al. An epigenetic biomarker of aging for lifespan and healthspan. Aging (Albany NY) 10, 573-591 (2018).

77. Kresovich, J. K., Xu. Z., O'Brien. K. M., Weinberg. C. R., Sandler, D. P., Taylor, J. A. Methylation-based biological age and breast cancer risk. J. Natl Cancer Inst. 111, 1051-1058 (2019).

78. Johnson, R. H., Chien, F. L. \& Bleyer, A. Incidence of breast cancer with distant involvement among women in the United States, 1976 to 2009. JAMA 309, 5 (2013).

79. Fidler, M. M., Gupta, S., Soerjomataram, I., Ferlay, J., Steliarova-Foucher, E. \& Bray, F. Cancer incidence and mortality among young adults aged $20-39$ years worldwide in 2012: a population-based study. Lancet Oncol. 18, 1579-1589 (2017).

80. Cho, Y. H., Yazici, H., Wu, H. C., Terry, M. B., Gonzalez, K., Qu, M. et al. Aberrant promoter hypermethylation and genomic hypomethylation in tumor, adjacent normal tissues and blood from breast cancer patients. Anticancer Res. 30, 24892496 (2010).

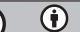

Open Access This article is licensed under a Creative Commons Attribution 4.0 International License, which permits use, sharing, adaptation, distribution and reproduction in any medium or format, as long as you give appropriate credit to the original author(s) and the source, provide a link to the Creative Commons license, and indicate if changes were made. The images or other third party material in this article are included in the article's Creative Commons license, unless indicated otherwise in a credit line to the material. If material is not included in the article's Creative Commons license and your intended use is not permitted by statutory regulation or exceeds the permitted use, you will need to obtain permission directly from the copyright holder. To view a copy of this license, visit http://creativecommons. org/licenses/by/4.0/.

(c) The Author(s) 2020 